\title{
Hemodynamic parameters predict adverse outcomes following biventricular conversion with single-ventricle palliation takedown
}

\author{
Melissa A. Herrin, BA, ${ }^{a}$ David Zurakowski, PhD,${ }^{b}$ Christopher W. Baird, MD, ${ }^{a}$ Puja Banka, MD,
} Jesse J. Esch, MD, MSc, ${ }^{\mathrm{c}}$ Pedro J. del Nido, MD, ${ }^{\mathrm{a}}$ and Sitaram M. Emani, MD

\begin{abstract}
Objective: Patients with a borderline left ventricular hypoplasia in the hypoplastic left heart syndrome variant or an unbalanced atrioventricular canal who undergo initial single-ventricle palliation may be candidates for biventricular (BiV) conversion following left ventricle (LV) recruitment procedures. We investigated associations among preoperative parameters and postoperative outcomes in patients undergoing $\mathrm{BiV}$ conversion.
\end{abstract}

Methods: We performed a retrospective review of patients who underwent BiV conversion to determine variables associated with clinical outcomes. Predictor variables included cardiac diagnosis, age and weight, LV dimension, $L V$ end diastolic volume, LV mass, preoperative LV end diastolic pressure (LVEDP), and preoperative left atrial pressure. Primary outcome was a composite of death, heart transplant, or BiV takedown.

Results: Of 51 patients, 11 experienced primary outcome (22\%). Patients with hypoplastic left heart syndrome variant were more likely to experience primary outcome than those with an unbalanced atrioventricular canal $(30 \%$ vs $6 \% ; P=.03$ ). Receiver operating characteristic analysis demonstrated that preoperative LVEDP had good predictive accuracy in classifying patients with and without the primary outcome (area under the curve, 0.757; $95 \%$ confidence interval, 0.594-0.919; $P=.012$ ). The Youden $J$-index indicated a cutoff of LVEDP $\geq 13 \mathrm{~mm} \mathrm{Hg}$ as optimal for predicting the primary outcome. Multivariable Cox regression demonstrated that LVEDP $\geq 13 \mathrm{~mm} \mathrm{Hg}$ (adjusted hazard ratio, 4.00; $P=.037$ ) and postoperative right ventricle pressure $>3 / 4$ (adjusted hazard ratio, 21.75; $P<.001$ ) were significantly associated with primary outcome, independent of age, weight, and diagnosis.

Conclusions: Elevated preoperative LVEDP is a risk factor for suboptimal postoperative hemodynamic parameters and adverse outcome following $\mathrm{BiV}$ conversion from single-ventricle palliation. (J Thorac Cardiovasc Surg 2017;154:572-82)

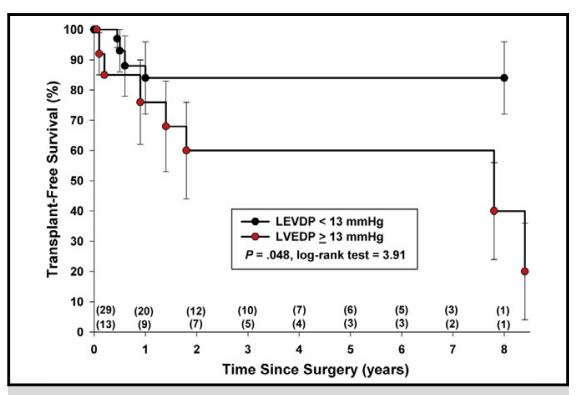

Freedom from death, transplant, and biventricular (BiV) takedown: Preoperative left ventricular end diastolic pressure (LVEDP) $<13 \mathrm{~mm} \mathrm{Hg}$ versus $\geq 13 \mathrm{~mm} \mathrm{Hg}$.

\section{Central Message}

Preoperative elevated left ventricular end diastolic pressure is associated with adverse events following biventricular conversion.

\section{Perspective}

We found that elevated preoperative left ventricular end diastolic pressure was associated with adverse events and suboptimal hemodynamic parameters in patients with borderline left heart following biventricular (BiV) conversion. This study will inform clinical management of patients who have undergone single-ventricle palliation and left ventricle recruitment who may be candidates for $\mathrm{BiV}$ conversion.

See Editorial Commentary page 583.

See Editorial page 570.
From the Departments of ${ }^{\mathrm{a} C}$ ardiac Surgery, ${ }^{\mathrm{b}}$ Anesthesia and Surgery, and ${ }^{\mathrm{c}}$ Cardiology, Boston Children's Hospital, Harvard Medical School, Boston, Mass.

Read at the 96th Annual Meeting of The American Association for Thoracic Surgery, Baltimore, Maryland, May 14-18, 2016.

Received for publication May 18, 2016; revisions received Jan 18, 2017; accepted for publication Feb 13, 2017; available ahead of print May 16, 2017.

Address for reprints: Sitaram M. Emani, MD, Department of Cardiac Surgery, Boston Children's Hospital, 300 Longwood Ave, Bader 273, Boston, MA 02115 (E-mail: Sitaram.Emani@cardio.chboston.org).

$0022-5223 / \$ 36.00$

Copyright $(\subset) 2017$ by The American Association for Thoracic Surgery

http://dx.doi.org/10.1016/j.jtcvs.2017.02.070
Patients with borderline left ventricular (LV) hypoplasia, including those with borderline hypoplastic left heart syndrome (bHLHS) and right-dominant atrioventricular canal

Scanning this QR code will take you to the article title page. To view the AATS 2016 Webcast, see the URL next to the video thumbnail. 


$$
\begin{aligned}
& \text { Abbreviations and Acronyms } \\
& \begin{aligned}
\text { ASD } & =\text { atrial septal defect } \\
\text { BiV } & =\text { biventricular } \\
\text { bHLHS } & \text { borderline hypoplastic left heart } \\
& \text { syndrome } \\
\text { EFE } & \text { endocardial fibroelastosis } \\
\text { LAp } & =\text { left atrial pressure } \\
\text { LV } & =\text { left ventricle } \\
\text { LVD } & =\text { left ventricle dimension } \\
\text { LVEDP } & \text { left ventricular end diastolic pressure } \\
\text { ROC } & =\text { receiver operating characteristic } \\
\text { RVP } & =\text { right ventricular pressure } \\
\text { SBP } & =\text { systolic blood pressure } \\
\text { SLVR } & =\text { staged left ventricular recruitment } \\
\text { SVP } & =\text { single-ventricle palliation } \\
\text { uAVC } & =\text { right-dominant unbalanced } \\
& \text { atrioventricular canal }
\end{aligned}
\end{aligned}
$$

(uAVC) defects, commonly undergo single-ventricle palliation (SVP). Patients with certain anatomic features (ie, valve regurgitation, ventricular dysfunction, pulmonary vascular disease, or parenchymal disease) experience poor long-term outcomes following SVP. ${ }^{1-5}$

Certain patients who have undergone SVP are candidates for staged LV recruitment (SLVR), a strategy in which procedures to relieve inflow and outflow tract obstruction and promote blood flow through the LV eventually lead to growth of left heart structures. SLVR allows decisions regarding long-term SVP or biventricular (BiV) circulation to be delayed until several years of age, based on response of the left heart to recruitment maneuvers. Patients can remain with SVP if LV growth is determined to be inadequate for BiV conversion. SLVR has been shown to result in normalization of left heart size, improve the ability of the LV to function as the systemic ventricle, and has allowed conversion to $\mathrm{BiV}$ circulation in a subset of patients. ${ }^{6}$ However, patients undergoing $\mathrm{BiV}$ conversion following SLVR are at risk for perioperative mortality, pulmonary hypertension, LV systolic and diastolic dysfunction, and valve dysfunction. ${ }^{6,7}$

Criteria for patient selection for $\mathrm{BiV}$ conversion following SLVR have not been established, but are typically based on assessment of imaging and hemodynamic parameter data. Size and function of left heart structures (ie, LV, aortic valve, and mitral valve) are typically used as determinants of candidacy for BiV conversion. However, the ability of hemodynamic and echocardiographic parameters to predict long-term outcomes of $\mathrm{BiV}$ conversion has not been established..$^{7-10}$ The purpose of this study was to describe a single institution experience with biventricular conversion and investigate the association between preoperative echocardiographic and pre- and postoperative hemodynamic parameters and postoperative outcomes in pediatric patients who underwent BiV conversion following SVP.

\section{PATIENTS AND METHODS}

This study was a retrospective review of patients with a diagnosis of right-dominant uAVC or bHLHS who underwent initial SVP at any center followed by eventual BiV conversion at Boston Children's Hospital between January 2003 and November 2015. The study design and methods were approved by the Boston Children's Hospital Institutional Review Board.

Patients were included in our analysis if they were diagnosed with bHLHS or right-dominant uAVC and underwent stage I palliation with either aortopulmonary amalgamation (Damus-Kaye-Stansel procedure) or hybrid approach in infancy, subsequently underwent SLVR procedures, and underwent eventual conversion from single-ventricle to BiV circulation. The population with bHLHS who underwent SLVR have been defined previously, and included patients with Shones complex or hypoplastic left heart syndrome variants without aortic or mitral atresia. ${ }^{6}$ Patients were excluded if they underwent initial SVP followed by BiV conversion for other diagnoses; that is, complex double-outlet right ventricle or transposition of the great arteries.

Patients were selected to undergo BiV conversion based on imaging studies demonstrating favorable increase in the size of left heart structures (ie, LV, aortic valve, and mitral valve) following initial palliation either with or without adjunctive SLVR procedures. BiV conversion involved reversal of Damus-Kaye-Stansel procedure or hybrid circulation, aortopulmonary shunts, cavopulmonary shunt, or Fontan baffles. Re-establishment of separate LV to aorta and right ventricle to pulmonary artery continuity was achieved by either direct reanastomosis or Ross procedure.

The following data were collected from review of preoperative imaging and medical records: gender, age, weight, and underlying cardiac diagnosis. Preoperative echocardiographic parameters (ie, LV end diastolic volume absolute value and $z$ score, LV long axis dimension [LVD] absolute value and $z$ score, and LV mass absolute value and $z$ score), and catheterization data (ie, mean left atrial pressure [LAp] and LV end diastolic pressure [LVEDP]) were extracted from the most recent studies before BiV conversion. Operative details at stage I procedure, staged recruitment procedures, and $\mathrm{BiV}$ conversion were collected from the operative notes.

Postoperative echocardiogram and catheterization data were collected from the most recent study for most patients. However, for patients who died, underwent transplantation, or underwent $\mathrm{BiV}$ conversion takedown, data were collected from the most recent catheterization or echocardiogram immediately before an adverse event. From catheterization reports, postoperative hemodynamic parameters, including LAp, LVEDP, and systolic right ventricular pressure (RVP) were collected. From the echocardiogram, LV end diastolic volume, LV ejection fraction, and estimated RVP from tricuspid regurgitation jet were collected. When RVP was not available on catheterization study, the value obtained from echocardiogram was used. RVP was expressed as a percentage of systolic blood pressure measured simultaneously.

Postoperative clinical outcomes, including surgical or catheter-based reinterventions, cardiac transplantation, and mortality were recorded. Time to event was calculated. Clinical follow-up was calculated as the time between $\mathrm{BiV}$ conversion procedure and the latest recorded clinical encounter in the electronic medical record.

Primary outcome was a composite end point consisting of death, heart transplant (transplant), and BiV takedown to SVP (takedown). We used multivariable logistic regression and Cox regression modeling with timedependent covariates to determine which predictor variables were significantly associated with primary outcome. Variables entered into the analysis included diagnosis, age and weight at time of surgery, LVD $z$ score, LV end 
diastolic volume $z$ score, LV mass $z$ score, preoperative LVEDP, and preoperative mean LAp.

\section{Statistical Analysis}

Data were described as frequency (\%) for categorical variables and as median (interquartile range [IQR]) for continuous variables. Univariate analysis comparing patient demographic data, echocardiographic, catheterization, and procedure data were compared using $\chi^{2}$, Fisher exact test, or Mann-Whitney $U$ test, as appropriate. Univariate analyses between patient outcomes and diagnosis were evaluated by the likelihood ratio test in logistic regression. All variables attaining statistical significance by univariate analysis $(P<.05)$ were then tested by multivariable Cox proportional hazards regression to identify independent predictors of primary outcome and to determine adjusted hazard ratios and $95 \%$ confidence intervals (CIs). Cox proportional-hazards regression modeling with a time-dependent covariate was applied to evaluate the effect of RVP:systolic blood pressure (SBP) at the multiple catheterization procedures within the same patient on the primary outcome using the coxph function with exact treatment of ties and a start-stop setup to create intervals of time in R software (R Foundation for Statistical Computing, Vienna, Austria). ${ }^{11}$

Receiver operating characteristic (ROC) curves were constructed to determine diagnostic thresholds associated with primary outcome with the Youden $J$-index to identify optimal cutoff values. ${ }^{12}$ The Pearson correlation coefficient $(r)$ was calculated to measure linear association between preoperative and postoperative LAp. Survival without transplant or takedown to SVP was analyzed for the cohort using the Kaplan-Meier product-limit method with the Greenwood formula to derive $95 \%$ CIs around the curves and the log-rank test was used to compare groups. ${ }^{13}$ All statistical analyses were performed with IBM SPSS Statistics (version 23.0; IBM-SPSS Inc, Armonk, NY).

TABLE 1. Characteristics of 51 patients undergoing biventricular (BiV) conversion with Damus-Kaye-Stansel (DKS) or hybrid stage I takedown: Demographic characteristics, recruitment pathways, operative interventions employed during BiV conversion, and postoperative interventions

\begin{tabular}{|c|c|c|}
\hline Variable & bHLHS $(n=33)$ & $\mathbf{u A V C}(\mathbf{n}=\mathbf{1 8})$ \\
\hline \multicolumn{3}{|l|}{ Demographic characteristic } \\
\hline Age (y) & $2.0(0.7-4.7)$ & $2.8(0.6-4.3)$ \\
\hline Weight (kg) & $12(6.9-15.7)$ & $11.2(6.0-14.8)$ \\
\hline Male gender & $19(58)$ & $10(56)$ \\
\hline \multicolumn{3}{|l|}{ Stage I palliative procedure } \\
\hline DKS & $28(85)$ & $15(83)$ \\
\hline Hybrid & $5(15)$ & $3(17)$ \\
\hline \multicolumn{3}{|l|}{ Procedures performed at staged left ventricle recruitment } \\
\hline EFE resection & $14(42)$ & $0(0)$ \\
\hline MVR/AVVR & $13(39)$ & $1(6)$ \\
\hline AoVR & $12(36)$ & $0(0)$ \\
\hline ASD restriction & $17(52)$ & $4(22)$ \\
\hline \multicolumn{3}{|l|}{ Catheterization procedures performed at staged left ventricle recruitment } \\
\hline ASD creation/balloon dilation & $14(42)$ & $0(0)$ \\
\hline Aortic valve intervention & $15(45)$ & $0(0)$ \\
\hline \multicolumn{3}{|l|}{ Operative interventions at time of $\mathrm{BiV}$ conversion } \\
\hline Takedown bidirectional Glenn procedure & $17(52)$ & $11(61)$ \\
\hline Takedown Fontan & $5(15)$ & $2(11)$ \\
\hline AoVR & $9(27)$ & $2(11)$ \\
\hline Ross procedure & $7(21)$ & $0(0)$ \\
\hline ASD closure & $11(33)$ & $11(61)$ \\
\hline Ventricular septal defect closure & $10(30)$ & $8(44)$ \\
\hline EFE resection & $9(27)$ & $0(0)$ \\
\hline MVR & $14(42)$ & $3(17)$ \\
\hline Right ventricle-to-pulmonary artery shunt or systemic-to-pulmonary artery shunt & $11(33)$ & $2(11)$ \\
\hline \multicolumn{3}{|l|}{ Post-BiV conversion interventions } \\
\hline EFE resection & $2(6)$ & $0(0)$ \\
\hline MVR & $13(39)$ & $8(44)$ \\
\hline AoVR & $6(18)$ & $0(0)$ \\
\hline Ross procedure & $2(6)$ & $0(0)$ \\
\hline Tricuspid valve repair or replacement & $0(0)$ & $1(6)$ \\
\hline Pacemaker placement & $5(15)$ & $1(6)$ \\
\hline Pulmonary artery plasty & $0(0)$ & $2(11)$ \\
\hline Subaortic resection & $1(3)$ & $4(22)$ \\
\hline Pulmonary valve repair or replacement & $0(0)$ & $1(6)$ \\
\hline Systemic vein dilation/stent & $0(0)$ & $2(11)$ \\
\hline Fenestrated ASD patch & $2(6)$ & $2(11)$ \\
\hline
\end{tabular}

Values are presented as median (interquartile range) or n (\%). bHLHS, Borderline hypoplastic left heart syndrome; $u A V C$, right-dominant complete atrioventricular canal; $D K S$, Damus-Kaye-Stansel; EFE, endocardial fibroelastosis; $M V R$, mitral valve repair/replacement; $A V V R$, atrioventricular valve repair; $A o V R$, aortic valve repair/replacement; $A S D$, atrial septal defect; $B i V$, biventricular. 


\section{RESULTS \\ Patient Characteristics}

Fifty-one patients met inclusion criteria and underwent BiV conversion between January 2003 and November 2015. Median age at BiV conversion was 2.7 years (IQR, 0.6-4.6 years) and median weight was $11.9 \mathrm{~kg}$ (IQR, 6.6$15.4 \mathrm{~kg}$ ). Patient characteristics are shown in Table 1. One or more risk factors for SVP was present in 38 out of 51 $(75 \%)$ patients.

\section{Operative Interventions at Staged Recruitment and Biventricular Conversion}

Recruitment procedures for the LV were typically performed concomitantly at the time of staged palliative procedures or as a separate procedure if a patient had undergone previous SVP. Recruitment procedures were performed during stage I palliation in 4 patients $(8 \%)$, at bidirectional Glenn surgery in 10 patients $(20 \%)$, at Fontan in 6 patients $(12 \%)$, or at a separate procedure following the bidirectional Glenn surgery in 13 patients (25\%) (Table 1). Median time between first recruitment procedure and $\mathrm{BiV}$ conversion was 1.3 years (IQR, 0.6-2.3 years). Median hospital length of stay following $\mathrm{BiV}$ conversion was 25 days (IQR, 12-38 days). Operative and catheter-based interventions performed for SLVR, as well as technical details of $\mathrm{BiV}$ conversion, including concomitant valvular procedures and management of atrial septal defect (ASD), are described in Table 1.

\section{Postoperative Outcomes}

Median follow-up time after $\mathrm{BiV}$ conversion was 2.7 years (range, 0.2 months- 12 years). Eleven of 51 patients $(22 \%)$ experienced the primary outcome composite end point of death $(n=7)$, transplant $(n=3)$, or takedown $(\mathrm{n}=1)$. Following BiV conversion, freedom from death, transplant, or takedown to SVP was $80 \%$ and $73 \%$ at 3 and 5 years, respectively (Figure 1). Median time to death following $\mathrm{BiV}$ conversion was 351 days (range, 733091 days), median time to transplant listing was 233 days (range, 55-411 days), median time to transplant was 237 days (range, 158-2828 days), and time to BiV conversion takedown in 1 patient was 84 days.

Of the 7 patients who died, 1 patient was placed on extracorporeal membrane oxygenation while awaiting transplant with course complicated by acute kidney injury and liver dysfunction. One patient with left atrial hypertension, pulmonary hypertension, and diastolic dysfunction died following ventricular assist device bridge to transplant. One patient died in the hospital before discharge after $\mathrm{BiV}$ surgery in the setting of renal failure requiring continuous venovenous hemodialysis, paralytic ileus, and gramnegative peritonitis. Another patient died from respiratory distress in the setting of pneumonia and sepsis after being

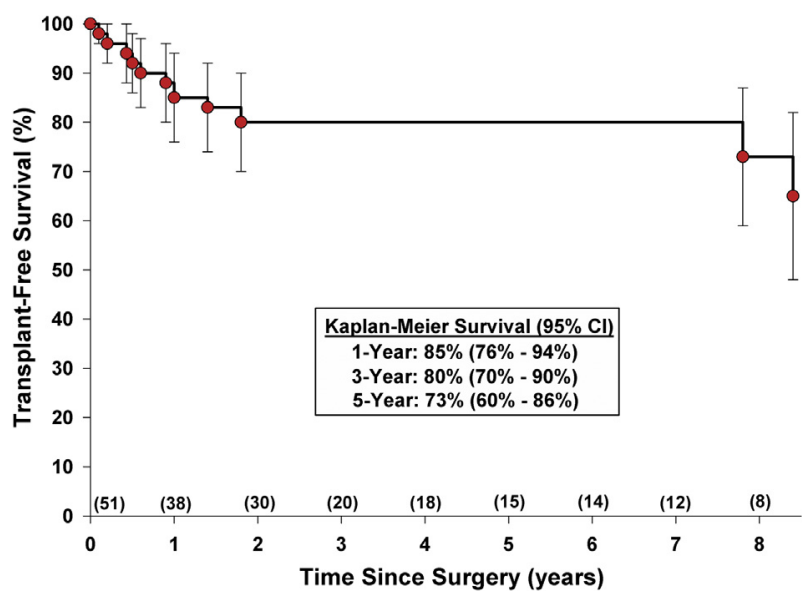

FIGURE 1. Kaplan-Meier curve of freedom from death, cardiac transplant, and surgical biventricular takedown to single-ventricle palliation in 51 patients undergoing biventricular conversion following stage I procedure. Estimated transplant-free survival at 3 years is $80 \%(95 \%$ confidence interval, $70 \%-90 \%$ ). $C I$, Confidence interval.

discharged from BiV hospitalization. Most had documented elevated right-sided pressures, pulmonary edema, left atrial hypertension, and/or LV diastolic dysfunction before decline.

Of 3 patients who underwent cardiac transplant, 1 patient underwent mitral valve repair and aortic valve repair 6 years after $\mathrm{BiV}$ conversion. This patient had progressive LV dysfunction, diastolic then systolic, with low output state and evidence of pulmonary hypertension and right ventricle dysfunction. The patient was listed for transplant and underwent left ventricular assist device implantation 4 months after mitral valve repair or replacement or aortic valve repair or replacement. The patient underwent transplant approximately 1 year following left ventricular assist device placement. The second patient had a Berlin biventricular assist device implantation 6 months following $\mathrm{BiV}$ conversion in the setting of severe LV dysfunction. This patient eventually required extracorporeal membrane oxygenation cannulation and underwent transplant 1.5 months following biventricular assist device implantation. The third patient exhibited signs of left atrial hypertension and frequent hospitalizations for signs of heart failure and for gastrointestinal and pulmonary symptoms. The patient returned home from Boston 5 months following BiV conversion and underwent transplant shortly after returning.

Ten of 11 patients who reached primary outcome experienced RVP $>3 / 4 \quad$ SBP before the primary end point, and postoperative catheterization report was not available for the other patient reaching primary outcome. The median time to study that demonstrated development of elevated RVP among those 10 patients with a primary event was 8 months (IQR, 3-35 months). Median time from the first study that demonstrated elevated RVP until primary event was 6 months (IQR, 3-8 months). 
Surgical reintervention following BiV conversion was required in 25 patients $(49 \%)$. Reoperation for mitral valve repair or replacement was performed in 11 bHLHS and 8 uAVC patients. Aortic valve repair or replacement was performed in $6(18 \%)$ patients with bHLHS, including 2 Ross procedures. Two patients with bHLHS underwent reoperation for further endocardial fibroelastosis (EFE) resection. Tricuspid valve repair was performed in 1 patient $(6 \%)$ and pulmonary artery plasty was performed in 2 patients $(11 \%)$ with uAVC. Permanent pacemaker systems were placed for heart block in 1 patient $(2 \%)$ and for resynchronization in 5 patients $(10 \%)$; subaortic resection was performed in 5 patients $(10 \%)$; fenestrated atrial septal defect patch was performed in 4 patients $(8 \%)$; and systemic vein dilation/stent was performed in 2 patients (4\%).

\section{Preoperative Variables Associated With Postoperative Outcomes}

Preoperative echocardiographic parameters were compared between patients with and without the primary composite end point. By univariate analysis, age, weight, gender, and $z$ scores for LVD, LV end diastolic volume, and LV mass were not statistically different between those who experienced a primary composite end point compared with those who did not (Table 2). Similarly, preoperative LAp measured at cardiac catheterization was not significantly different between the 2 groups.

To ascertain whether diagnosis (bHLHS vs uAVC) was associated with the primary outcome, we compared number and percentage of patients with each diagnosis who reached primary outcome. Of 11 patients who reached primary outcome, 10 had a diagnosis of bHLHS, and 1 had a diagnosis of uAVC (Table 2). Ten of 33 patients with bHLHS (30\%) compared with only 1 of 18 patients with uAVC $(6 \%)$ had the primary outcome (odds ratio, $7.4 ; P=.03$ ) (Table 2). This suggests that the risk of an adverse outcome following $\mathrm{BiV}$ conversion is significantly elevated in patients with a diagnosis of bHLHS compared with uAVC.
Median preoperative LVEDP was significantly greater among those who reached primary composite end point after $\mathrm{BiV}$ conversion (13 $\mathrm{mm} \mathrm{Hg}$; IQR, $12-16 \mathrm{~mm} \mathrm{Hg}$ ) compared with those who did not $(11 \mathrm{~mm} \mathrm{Hg}$; IQR, 10$12 \mathrm{~mm} \mathrm{Hg})(P=.01)$ (Table 2). ROC analysis of LVEDP demonstrated that preoperative LVEDP had good predictive accuracy in classifying patients with and without the primary outcome (area under the curve, $0.757 ; 95 \% \mathrm{CI}$, $0.594-0.919 ; P=.012$ ). The Youden $J$-index indicated a cutoff of LVEDP $\geq 13 \mathrm{~mm} \mathrm{Hg}$ as optimal for predicting the primary outcome and this provided sensitivity of $64 \%$, and specificity of $81 \%$ ). Multivariable Cox proportional hazards regression analysis confirmed that a preoperative LVEDP $\geq 13 \mathrm{~mm} \mathrm{Hg}$ was significantly associated with the primary outcome (odds ratio, $4.0 ; 95 \%$ CI, 1.17-15.09; $P=.037$ ) after adjusting for age, weight, and diagnosis (Table 3).

Multivariable Cox proportional hazards regression analysis confirmed that a preoperative LVEDP $>=13 \mathrm{~mm} \mathrm{Hg}$ was significantly associated with the primary outcome (odds ratio, 4.0; 95\% CI, 1.17-15.09; $P=.037$ ) after adjusting for age, weight, and diagnosis (Table 3). Log-rank testing indicated a significant difference transplant-free survival in patients with preoperative LVEDP $<13 \mathrm{~mm} \mathrm{Hg}$ compared with LVEDP $>=13 \mathrm{~mm} \mathrm{Hg}(P=.048$; logrank test $=3.91)($ Figure 2$)$.

\section{Postoperative Variables Associated With Primary Outcome}

Median postoperative RVP:SBP was significantly greater (0.92; IQR, 0.85-1.1) among those who experienced a primary outcome compared with those who did not $(0.63$; IQR, 0.52-0.74) $(P<.001)$. Multivariable Cox regression analysis demonstrated that postoperative RVP $>3 / 4$ SBP was significantly associated with primary outcome end point of death, transplant, or takedown after adjustment for covariates of age, weight, and diagnosis (hazard ratio, 21.75; 95\% CI, 2.57-184.22; $P<.001$ ) (Table 4). We

TABLE 2. Preoperative demographic, echocardiographic, and catheterization parameters in patients experiencing the primary outcome compared with those without a primary outcome

\begin{tabular}{lccc}
\hline \multicolumn{1}{c}{ Preoperative variable } & Primary outcome $(\mathbf{n}=\mathbf{1 1})$ & No primary outcome $(\mathbf{n}=\mathbf{4 0})$ & $\boldsymbol{P}$ value \\
\hline bHLHS & $10(91)$ & $23(58)$ & $.03 *$ \\
Male gender & $6(55)$ & $23(58)$ & 1 \\
Age $(\mathrm{y})$ & $2.7(0.5$ to 4.6$)$ & $11.8(6.6$ to 4.7$)$ & $15.2)$ \\
Weight $(\mathrm{kg})$ & $12(6.6$ to 15.6$)$ & $-0.42(-1.73$ to 0.51$)$ & .94 \\
LV dimension $z$ score & $-1.2(-2.7$ to 1.2$)$ & $-0.72(-1.9$ to 0.16$)$ & .98 \\
LVEDV $z$ score & $-0.83(-1.9$ to 1.2$)$ & $-0.61(-2.0$ to 1.0$)$ & .65 \\
LV mass $z$ score & $-0.56(-2.1$ to 0.6$)$ & $12(8$ to 13$)$ & .97 \\
Preoperative left atrial pressure $(\mathrm{mm} \mathrm{Hg})$ & $13(9$ to 14$)$ & $11(10$ to 12$)$ & .66 \\
Preoperative LVEDP $(\mathrm{mm} \mathrm{Hg})$ & $13(12$ to 16$)$ & .26 \\
\hline
\end{tabular}

Values are presented as $\mathrm{n}(\%)$ or median (interquartile range). $b H L H S$, Borderline hypoplastic left heart syndrome; $L V$, left ventricle; $L V E D V$, left ventricular end diastolic volume; LVEDP, left ventricular end diastolic volume. *Statistically significant. 
TABLE 3. Multivariable Cox proportional hazards regression analyzing left ventricular end diastolic volume (LVEDP) as a predictor of primary outcome adjusting for patient characteristics

\begin{tabular}{lcc}
\hline $\begin{array}{c}\text { Parameter associated with } \\
\text { primary outcome (death, } \\
\text { transplant, or takedown) }\end{array}$ & $\begin{array}{c}\text { Adjusted hazard } \\
\text { ratio }(\mathbf{9 5} \% \text { confidence } \\
\text { interval) }\end{array}$ & P value \\
\hline Age (y) & $0.81(0.38-1.77)$ & .604 \\
Weight $(\mathrm{kg})$ & $0.96(0.69-1.34)$ & .816 \\
Diagnosis: bHLHS vs uAVC & $2.76(0.32-24.16)$ & .309 \\
Preoperative LVEDP $\geq 13 \mathrm{~mm} \mathrm{Hg}$ & $4.00(1.17-15.09)$ & $.037^{*}$ \\
\hline
\end{tabular}

bHLHS, Borderline hypoplastic left heart syndrome; $u A V C$, right-dominant complete atrioventricular canal; $L V E D P$, left ventricular end diastolic volume. *Statistically significant.

when we applied Cox proportional-hazards regression modeling with a time-dependent covariate, results indicated a significant effect of a higher RVP:SBP on risk of the primary outcome in patients with biventricular repair (hazard ratio, $112.4 ; P=.0056)$.

Median postoperative $\mathrm{LAp}(\mathrm{mm} \mathrm{Hg})$ at follow-up cardiac catheterization was significantly greater (21.5; IQR, 19.526.5) among those who experienced a primary composite end point compared with those who did not (17.5; IQR, 12.5-23.8) $(P=.037)$, but did not remain significant on multivariable analysis.

\section{Comments}

This study describes outcomes of patients with bHLHS and uAVC undergoing BiV conversion following SVP and $\mathrm{LV}$ recruitment procedures and investigated the risk factors for death, transplant, and takedown to SVP following BiV conversion. We found that underlying diagnosis of bHLHS was associated with primary outcomes. Furthermore, independent of age, weight, gender, and diagnosis, preoperative

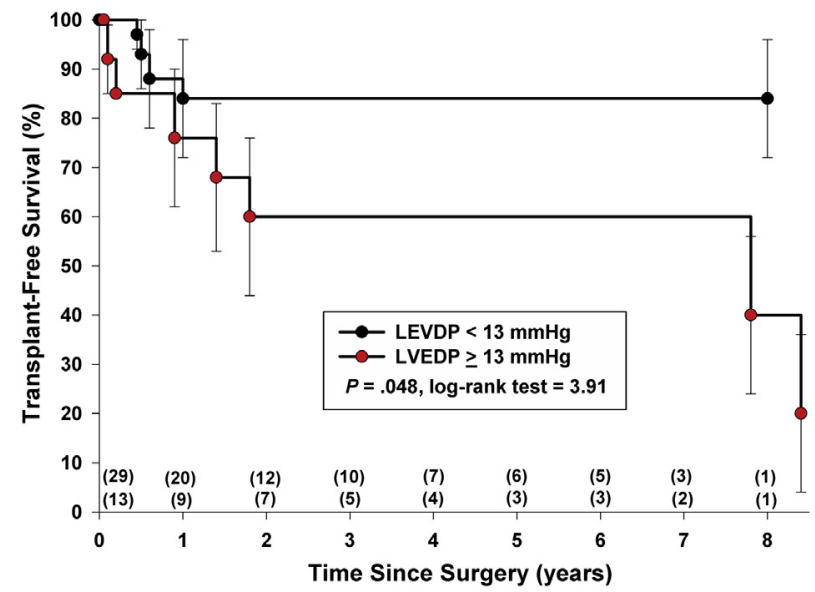

FIGURE 2. Kaplan-Meier curves comparing freedom from death, cardiac transplant, and biventricular takedown in patients with preoperative left ventricular end diastolic pressure $($ LVEDP $)<13 \mathrm{~mm} \mathrm{Hg}$ compared with LVEDP $\geq 13 \mathrm{~mm} \mathrm{Hg}$. Log-rank testing indicated significant difference between the 2 groups $(P=.048$; log-rank test $=3.91)$.
TABLE 4. Multivariable Cox proportional hazards regression analyzing right ventricle pressure (RVP) as a predictor of primary outcome, adjusting for patient characteristics

\begin{tabular}{lcc}
\hline $\begin{array}{c}\text { Parameter associated with } \\
\text { primary outcome (death, } \\
\text { transplant, or takedown) }\end{array}$ & $\begin{array}{c}\text { Adjusted hazard } \\
\text { ratio }(\mathbf{9 5} \% \text { confidence } \\
\text { interval) }\end{array}$ & P value \\
\hline Age $(\mathrm{y})$ & $1.38(0.62-3.11)$ & .439 \\
Weight $(\mathrm{kg})$ & $0.80(0.55-1.18)$ & .262 \\
Diagnosis: bHLHS vs uAVC & $4.51(0.47-43.43)$ & .142 \\
Postoperative RVP $>3 / 4$ systemic & $21.75(2.57-184.22)$ & $<.001^{*}$ \\
\hline
\end{tabular}

$b H L H S$, Borderline hypoplastic left heart syndrome; $u A V C$, right-dominant complete atrioventricular canal; $R V P$, right ventricle pressure. *Statistically significant.

LVEDP was predictive of adverse postoperative outcome, and preoperative LVEDP of 13 was a discriminator by ROC analysis.

Size of left heart structures (LV size and volume) as assessed by preoperative echocardiogram was not associated with primary outcome by statistical analysis. Although our findings were consistent with previous report by Mery and colleagues, ${ }^{14}$ other studies have demonstrated that size of the left heart area correlates with outcomes following primary $\mathrm{BiV}$ repair for patients with obstructive or hypoplastic LV. ${ }^{10,15}$ Whereas previous reports have examined the effects of size upon primary BiV repair, our study examined a cohort of patients undergoing BiV conversion after previous SVP, with a subset having undergone documented SLVR procedures. The advantage of the SLVR strategy is that it allows selection of favorable candidates for $\mathrm{BiV}$ conversion. In this series, increase in the size of the left heart structures was used as a selection criteria for $\mathrm{BiV}$ conversion; consequently, size of left heart structures did not emerge as significant predictor for outcomes. With this approach, decision regarding $\mathrm{BiV}$ conversion can be delayed until there has been adequate growth of the left heart structures, whereas patients with persistent hypoplasia despite SLVR are treated with a SVP strategy. This approach is an alternative in patients with risk factors for SVP, as demonstrated by the majority of patients in this series. Yet in patients without risk factors for SVP, the risk of $\mathrm{BiV}$ conversion must be carefully weighed against the longterm outcomes of SVP.

We found that median preoperative LVEDP was significantly greater among those who experienced primary outcome (ie, death, transplant, or takedown) compared with those who did not. Furthermore, patients with LVEDP $\geq 13 \mathrm{~mm} \mathrm{Hg}$ have a 7 times higher risk of primary outcome and/or RVP $>3 / 4$ SBP. Literature regarding the association between preoperative LVEDP and postoperative outcomes following $\mathrm{BiV}$ repair or conversion is limited, with many reporting hemodynamic parameters but not analyzing the associations among these parameters and outcomes. ${ }^{15-18}$ Elevated preoperative LVEDP is indicative of poor LV compliance despite adequate LV size, and suggests 
presence of abnormalities of myocardial relaxation or extracellular matrix composition. These abnormalities persist following $\mathrm{BiV}$ conversion and contribute to ongoing diastolic dysfunction, pulmonary artery hypertension, and ultimately adverse clinical outcomes. Status of the atrial septum and amount of pulmonary blood flow must be considered when interpreting the LVEDP data, because presence of an unrestrictive ASD may lead to low LVEDP due to inadequate LV filling. Many of the patients in this series had a restrictive ASD at the time of catheterization, allowing the LVEDP to serve as a reliable marker for ventricular compliance. Exuberant flow through aortopulmonary collaterals or Blalock-Taussig shunt may elevate the LVEDP, and thus LVEDP data must be correlated with flow across the mitral valve as measured by cardiac magnetic resonance imaging.

Compared with patients with uAVC, patients with bHLHS were significantly more likely to reach primary outcomes in multivariable analysis. Our findings suggest that a diagnosis of bHLHS is a risk factor for adverse outcomes after $\mathrm{BiV}$ conversion. Intrinsic left heart abnormalities have been well documented in patients with bHLHS. In addition to EFE, underlying myocardial fibrosis can result in in restrictive cardiomyopathy and poor contractility in bHLHS, and is distinctly absent in patients with uAVC. ${ }^{19,20}$ Such abnormalities may contribute to diastolic dysfunction, the extent and significance of which has been demonstrated in numerous previous studies of patients with bHLHS. ${ }^{21}$ Elevated LVEDP may also be a result of the left ventricular outflow tract obstruction in bHLHS. ${ }^{22}$

The association of bHLHS and elevated preoperative LVEDP with adverse outcomes after BiV conversion has several implications. Information regarding the presence of these preoperative risk factors may assist decision making and family counseling when considering BiV conversion. Options for patients with risk factors for poor outcome, particularly elevated LVEDP, include definitive SVP (completion Fontan), in patients without contraindications to SVP, ongoing observation, or re-evaluation of etiology for diastolic dysfunction. Rectifiable causes of diastolic dysfunction, including residual EFE, mitral regurgitation, or aortic valve stenosis or regurgitation, may require additional catheter-based or surgical intervention. Although surgical reoperation for the purpose of LV rehabilitation in this situation may seem aggressive, residual lesions can have a significant influence on LV compliance and the ability to attain eventual $\mathrm{BiV}$ conversion. In patients without obvious etiology for LV noncompliance, observation with hemodynamic parameter reassessment after 6 months may provide guidance regarding optimal long-term strategy. If compliance worsens with time, SVP may be preferable, whereas if the LVEDP decreases below 13, BiV conversion may be considered. In the absence of EFE or residual valvular disease, elevated LVEDP reflects myocardial diastolic dysfunction for which currently there is no effective medical or surgical treatment. Techniques to assess myocardial fibrosis, including late gadoliniumenhanced imaging ${ }^{23}$ and myocardial biopsy, ${ }^{24}$ and alternate methods to measure LV compliance may improve the ability to predict outcomes after BiV conversion. ${ }^{25}$ Ultimately, adjunctive therapies that lead to favorable myocardial remodeling are necessary to significantly increase efficacy of the SLVR strategy. ${ }^{26,27}$

We did not find a statistically significant association between preoperative LAp and primary outcome. Our finding is consistent with Banka and colleagues, ${ }^{15}$ who found no difference in preoperative left atrial pressures between transplant-free survivors and nonsurvivors with borderline bHLHS undergoing BiV repair. One possible explanation is that LAp in this cohort is a significant marker of mitral valve stenosis or regurgitation and not a reliable indicator of LV compliance in the presence of mitral valve disease. Mitral valve repair or replacement, frequently performed at the time of $\mathrm{BiV}$ conversion, may mitigate the effects of mitral valve pathology on long-term outcomes, thus leading to poor correlation between LAp and primary end points. The lack of statistical correlation found between preoperative LAp and postoperative LAp in this study further supports this explanation.

Elevated RVP following BiV conversion was predictive of adverse outcome. Elevated RVP may be an indicator of ongoing left heart dysfunction related to ventricular outflow tract obstruction, valvular dysfunction, and LV systolic and diastolic dysfunction. ${ }^{28}$ Previous studies have demonstrated the prognostic value of right ventricle dysfunction in cardiovascular disease in predicting morbidity and mortality. ${ }^{28,29}$ Elevated RVP may also impair LV function and cardiac output by contributing to leftward displacement of the interventricular septum. ${ }^{30}$ Elevated RVP may be independent of left heart hemodynamic parameters, but rather indicate primary abnormality of the pulmonary vasculature. Severe pulmonary hypertension is known to occur after $\mathrm{BiV}$ repair, even in patients with acceptable left heart function and hemodynamic parameters, and may be related to irreversible changes to the pulmonary vascular bed associated with diagnosis of bHLHS. ${ }^{10,16}$

Our findings suggest that RVP should be closely monitored following $\mathrm{BiV}$ conversion, and reversible causes of elevated RVP should be corrected. Investigation and early intervention for elevated RVP must be instituted before development of irreversible pulmonary vascular disease that may preclude cardiac transplantation or takedown to SVP. Catheterizations should be performed at regular (ie, annual) intervals so that adverse hemodynamic parameters may be addressed in patients who remain candidates for ventricular assist device placement, transplant, or takedown. 
Our study had several limitations. This was a retrospective review from a single institution with a small sample size subject to selection bias. Because the number of patients experiencing outcomes was small, the lack of statistical significance when testing for differences, especially in subgroup analysis, may have been due to lack of power. In addition, because patients were selected for BiV conversion only if they demonstrate near-normalization in size of left heart structures. This selection bias limits the ability of this study to assess the influence of left heart size on outcomes, yet suggests that sizes of left heart structures are important and supports using size to select patients for BiV conversion. Moreover, selection of patients to undergo BiV conversion, as well as preoperative assessment, imaging, and operative techniques, are evolving with experience over time. Lastly, unlike the application of the Cox regression model with fixed covariates, the use of time-dependent covariates must be interpreted with caution regarding any predictive time-toevent inferences because patients' exposures, health, and treatments change over time. In the case of RVP:SBP, the functional form of the covariate has the potential to introduce bias and the possibility of overfitting the data.

\section{CONCLUSIONS}

Elevated preoperative LVEDP is a risk factor for suboptimal postoperative hemodynamic parameters, including RVP $>3 / 4$ SBP, and adverse events in patients with bHLHS and uAVC after BiV conversion from SVP. Furthermore, we found that those patients with a preoperative LVEDP $<13 \mathrm{~mm} \mathrm{Hg}$ are less likely to experience unfavorable outcomes. This study expands upon previous literature identifying clinical parameters predictive of outcomes after $\mathrm{BiV}$ repair. Our results will aid in risk stratification and further inform selection of appropriate candidates for $\mathrm{BiV}$ conversion.

\section{Webcast}

You can watch a Webcast of this AATS meeting presentation by going to: http://webcast.aats.org/2016/Video/ Monday/05-16-16_Ballroom_III_1555_Herrin-800.mp4.

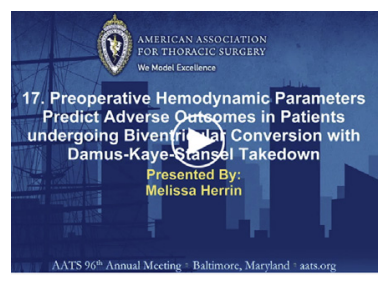

\section{Conflict of Interest Statement}

Authors have nothing to disclose with regard to commercial support.

This study was supported by funding from the Ethan Lindberg Foundation, Caledonia, IL.

\section{References}

1. Anderson PA, Sleeper LA, Mahony L, Colan SD, Atz AM, Breitbart RE, et al. Contemporary outcomes after the Fontan procedure: a Pediatric Heart Network multicenter study. J Am Coll Cardiol. 2008;52:85-98.

2. Barker PC, Nowak C, King K, Mosca RS, Bove EL, Goldberg CS. Risk factors for cerebrovascular events following fontan palliation in patients with a functional single ventricle. Am J Cardiol. 2005;96:587-91.

3. Camposilvan S, Milanesi O, Stellin G, Pettenazzo A, Zancan L, D'Antiga L. Liver and cardiac function in the long term after Fontan operation. Ann Thorac Surg. 2008;86:177-82.

4. Cohen MI, Rhodes LA. Sinus node dysfunction and atrial tachycardia after the Fontan procedure: the scope of the problem. Semin Thorac Cardiovasc Surg Pediatr Card Surg Annu. 1998;1:41-52.

5. Ohuchi H, Yasuda K, Miyazaki A, Kitano M, Sakaguchi H, Yazaki S, et al. Haemodynamic characteristics before and after the onset of protein losing enteropathy in patients after the Fontan operation. Eur J Cardiothorac Surg. 2013;43:e49-57.

6. Emani SM, McElhinney DB, Tworetzky W, Myers PO, Schroeder B, Zurakowski D, et al. Staged left ventricular recruitment after single-ventricle palliation in patients with borderline left heart hypoplasia. J Am Coll Cardiol. 2012;60:1966-74.

7. Hickey EJ, Caldarone CA, Blackstone EH, Lofland GK, Yeh T, Pizarro C, et al. Critical left ventricular outflow tract obstruction: the disproportionate impact of biventricular repair in borderline cases. J Thorac Cardiovasc Surg. 2007;134:1429-36.

8. Blaufox AD, Lai WW, Lopez L, Nguyen K, Griepp RB, Parness IA. Survival in neonatal biventricular repair of left-sided cardiac obstructive lesions associated with hypoplastic left ventricle. Am J Cardiol. 1998;82:1138-40. A1110.

9. Kovalchin JP, Brook MM, Rosenthal GL, Suda K, Hoffman JI, Silverman NH. Echocardiographic hemodynamic and morphometric predictors of survival after two-ventricle repair in infants with critical aortic stenosis. J Am Coll Cardiol. 1998;32:237-44.

10. Schwartz ML, Gauvreau K, Geva T. Predictors of outcome of biventricular repair in infants with multiple left heart obstructive lesions. Circulation. 2001;104:682-7.

11. Fisher LD, Lin DY. Time-dependent covariates in the Cox proportional-hazards regression model. Annu Rev Public Health. 1999;20:145-57.

12. Zhou X-H, McClish DK, Obuchowski NA. Statistical methods in diagnostic medicine. In: Wiley series in probability and statistics. 2nd ed. Hoboken: Wiley; 2011. 545.

13. Cox DR, Oakes D. Analysis of survival data. London: Chapman and Hall; 1984.

14. Mery CM, Nieto RM, De Leon LE, Morris SA, Zhang W, Colquitt JL, et al. The role of echocardiography and intracardiac exploration in the evaluation of candidacy for biventricular repair in patients with borderline left heart structures. Ann Thorac Surg. 2017;103:853-61.

15. Banka P, Schaetzle B, Komarlu R, Emani S, Geva T, Powell AJ. Cardiovascula magnetic resonance parameters associated with early transplant-free survival in children with small left hearts following conversion from a univentricular to biventricular circulation. J Cardiovasc Magn Reson. 2014;16:73.

16. Cavigelli-Brunner A, Bauersfeld U, Pretre R, Kretschmar O, Oxenius A, Valsangiacomo Buechel ER. Outcome of biventricular repair in infants with multiple left heart obstructive lesions. Pediatr Cardiol. 2012:33:506-12.

17. Hoashi T, Bove EL, Devaney EJ, Hirsch JC, Ohye RG. Outcomes of 1(1/2)- or 2 ventricle conversion for patients initially treated with single-ventricle palliation. J Thorac Cardiovasc Surg. 2011;141:419-24.

18. Kalish BT, Banka P, Lafranchi T, Tworetzky W, Del Nido P, Emani SM. Biventricular conversion after single ventricle palliation in patients with small left heart structures: short-term outcomes. Ann Thorac Surg. 2013;96:1406-12.

19. Feinstein JA, Benson DW, Dubin AM, Cohen MS, Maxey DM, Mahle WT, et al Hypoplastic left heart syndrome: current considerations and expectations. J Am Coll Cardiol. 2012;59:S1-42.

20. Mahmut N, Thompson M, Kinnear C, Shannon P, Chitayat D, Jaeggi E, Mital S Cellular senescence in hypoplastic left heart syndrome: young patients with old hearts. Circulation. 2008;118:S907-8.

21. Friedman KG, McElhinney DB, Rhodes J, Powell AJ, Colan SD, Lock JE, et al. Left ventricular diastolic function in children and young adults with congenital aortic valve disease. Am J Cardiol. 2013;111:243-9.

22. Colan SD, McElhinney DB, Crawford EC, Keane JF, Lock JE. Validation and re-evaluation of a discriminant model predicting anatomic suitability for biventricular repair in neonates with aortic stenosis. J Am Coll Cardiol. 2006; 47:1858-65.

23. Rathod RH, Prakash A, Powell AJ, Geva T. Myocardial fibrosis identified by cardiac magnetic resonance late gadolinium enhancement is associated with adverse ventricular mechanics and ventricular tachycardia late after Fontan operation. $J$ Am Coll Cardiol. 2010;55:1721-8. 
24. Mahtab EA, Gittenberger-de Groot AC, Vicente-Steijn R, Lie-Venema H, Rijlaarsdam ME, Hazekamp MG, et al. Disturbed myocardial connexin 43 and $\mathrm{N}$-cadherin expressions in hypoplastic left heart syndrome and borderline left ventricle. J Thorac Cardiovasc Surg. 2012;144:1315-22.

25. Pacher P, Nagayama T, Mukhopadhyay P, Batkai S, Kass DA. Measurement of cardiac function using pressure-volume conductance catheter technique in mice and rats. Nat Protoc. 2008;3:1422-34.

26. Tarui S, Sano S, Oh H. Stem cell therapies in patients with single ventricle physiology. Methodist Debakey Cardiovasc J. 2014;10:77-81.

27. Emani SM, Marx GR. Operations for improving left ventricular diastolic function. Curr Opin Cardiol. 2016;31:101-8.

28. Haddad F, Doyle R, Murphy DJ, Hunt SA. Right ventricular function in cardiovascular disease, part II: pathophysiology, clinical importance, and management of right ventricular failure. Circulation. 2008;117:1717-31.

29. Voelkel NF, Quaife RA, Leinwand LA, Barst RJ, McGoon MD, Meldrum DR, et al. Right ventricular function and failure: report of a National Heart, Lung, and Blood Institute working group on cellular and molecular mechanisms of right heart failure. Circulation. 2006;114: 1883-91.

30. Emani SM, del Nido PJ. Strategies to maintain biventricular circulation in patients with high-risk anatomy. Semin Thorac Cardiovasc Surg Pediatr Card Surg Annu. 2013;16:37-42.

Key Words: biventricular conversion, hypoplastic left heart syndrome

\section{Discussion}

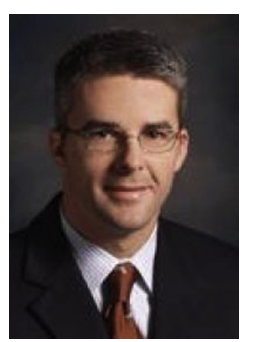

Dr Emmett D. McKenzie (Houston, Tex). I'd like to thank the Association for the privilege of discussing this and thank Ms Herrin for getting me a copy of the manuscript. It was beautifully written. I remind everybody that Ms Herrin is a medical student being mentored by the Boston Group, and a member for the day. Ms Herrin you should be proud.

The data you show here provide us with very useful information from a really well-designed retrospective study. The preoperative left ventricular end diastolic pressure $($ LVEDP) $>13$ portends a bad outcome. Preoperative LVEDP $\leq 11$ portends a good outcome.

Given how difficult it is to acquire these data in the catheterization laboratory and to trust these data depending on the volume-loading conditions, the heart rate, things like that, what are your recommendations about how we use this information clinically?

And the other question to follow-up to that is it seems to me that LVEDP is a pretty poor or crude indicator of left ventricle diastolic dysfunction, which is what the problem is. Is your group considering using the more sensitive way of measuring this, such as compliance measurements or conductance catheters?

Ms Herrin. Thank you, Dr McKenzie, for the questions. I will start with the second question first. We recognize that spot measurements of end diastolic pressure (EDP) can be highly variable. So we do some things to try to minimize variability when measuring EDP.
For starters, we record initial measurement at baseline before the patient has received volume so they are at euvolemic status.

Secondly, we perform provocative measures such as atrial septal defect occlusion during catheterization that might give us additional information as to how the left ventricle behaves.

And certainly, as you said, we are exploring the use of useful adjuncts such as conductance catheters.

With regard to your question about how LVEDP can be used clinically, was that what you asked?

Dr McKenzie. With a very small difference between the number that predicts a good outcome and the number that predicts a poor outcome, how do we use that practically in our daily lives?

Ms Herrin. Sure. So I think we would recommend a couple different options. Before biventricular conversion, as you said, we found a cutoff of 13. But as you said, there were poor outcomes even in patients that had 11 or 12 .

Anything in that range or above, first off might see if there are reversible causes for EDP such as in hypoplasts in particular, endocardial fibroelastosis (EFE) resection, valve repair, and valve disease.

And if not, then some of these patients may be able to be observed for some time and remeasured to see if the EDP might change. Certainly the benefit of remaining at single-ventricle palliation and using staged left ventricular recruitment is that there is always a possibility of going to biventricular conversion if preoperative hemodynamic parameters are more favorable.

Certainly if the patient remains at an elevated pressure, then we might consider the option that perhaps biventricular conversion should not be pursued and other options in particular.

Dr McKenzie. It seems obvious to me the problem is diastolic dysfunction afterward. The hypoplasts sort of stole the show as far as being the problem children. I could not tell from the manuscript, but it seemed like a large percentage of them or a large number of them have undergone EFE resection. Could you tell within the hypoplast group whether EFE resection is a predictor of poor outcome?

Ms Herrin. Yes, we did run that analysis. Among 11 outcomes, 10 were hypoplasts, and among those, 9 had undergone EFE resection. And we did find a significant association on $\chi^{2}$ analysis between having undergone EFE resection, biventricular conversion, and reaching a primary outcome. So it does seem to suggest that those patients who require EFE resection are at higher risk perhaps for compliance.

Dr McKenzie. Thank you. Congratulations again, great job.

Dr Jennifer C. Hirsch-Romano (Ann Arbor, Mich). Did you look at any of these patients who had undergone prior 
fetal interventions? Did that have an influence on their LVEDP at the time of biventricular conversion?

Ms Herrin. Thank you for the question. That is a good point. We did not run that analysis, although we could go back and analyze those data, and I appreciate that suggestion. It would be interesting.

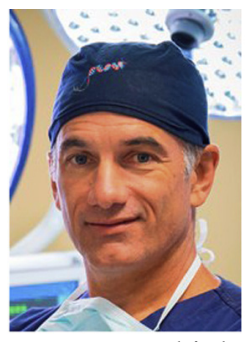

Dr Christopher Caldarone (Toronto, Ontario, Canada). That was a great presentation. The take-away message is liable to be an EDP of 13 is a critical decision point. I do not know whether that is your intention, but that may be the message many people take away.

Do you think that number might be different with regard to the unbalanced atrioventricular septal defect and the hypoplasts? Now, I know that you have a small sample size, and I know that it is hard to split your dataset, but in 1 group you have potential for EFE, but in the unbalanced atrioventricular septal defect group, the factors leading to a high EDP might be aortic valve insufficiency or kind of global dysfunction.

So should we be taking away the same cutpoint for both lesions, and do you think it is reasonable to lump them together?

Ms Herrin. Thank you for the question. Certainly we did see that our unbalanced canals did much better compared with our hypoplasts, and it may be reasonable to further investigate a different cutoff when we have more patients; that is, more data to analyze.

I will ask if Dr Emani wants to further comment on that.

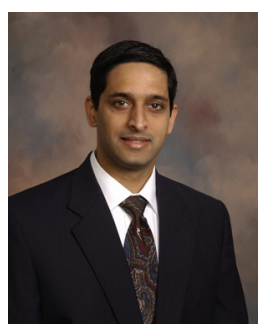

Dr Sitaram M. Emani (Boston, Mass). The multivariable regression analysis suggested that even patients with unbalanced atrioventricular canal who had unfavorable hemodynamic parameters had a poor outcome.

In my mind, LVEDP is certainly a sliding scale. I do not know that 13 is a magic number, but I start to get concerned when I see an LVEDP in the mid- to upper-teens whether it is an unbalanced canal or a hypoplastic left heart, and I would be cautious about jumping to a biventricular conversion. At that point it is an irreversible decision for at least a period of time, and my current preference is to wait it out or to look for the other reasons for LVEDP; for example, aortic valve regurgitation. I would even recommend an operation to see if I can ameliorate the regurgitation and see if that improves the EDPs before jumping to a biventricular conversion.

Dr Caldarone. So you might do 2 separate procedures, first fix the aortic valve insufficiency, see how things settle out, and then go back for a 2-ventricle repair?
Dr Emani. Yes. I know it seems aggressive, but in some situations I think we found that doing an operation to optimize outcomes is worthwhile when you take the entire picture into consideration.

Dr Caldarone. I think that is exactly what we would do, too.

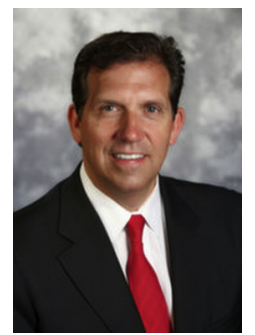

Dr Philip C. Smith (Akron, Ohio). Within the framework of the population of patients who had the more favorable numbers from the standpoint of converting the biventricular repair, did you look at the functional performance of these patients in the wake of the decision to go down that pathway? Avoiding transplant, death, or takedown is setting the bar low. How did these patients do after the decision to go down the pathway and the biventricular conversion was made?

Ms Herrin. Yes, thank you. That is an important question.

Those were not data that were specifically collected. We do have some follow-up on these patients, and it would be reasonable to go back and consult the documentation in the electronic medical record to see how they are doing. I do not know if Dr Emani has a sense for this clinically.

Dr Emani. Clearly these patients have more reinterventions. We are doing a lot to the mitral valves. We are working on the aortic valves, and at this point we prefer to repair rather than replace, and that means that we are frequently reoperating.

I do not know. Melissa, you can say our freedom from reintervention was in the $50 \%$ range at 5 years. So we are clearly back with these patients.

I think when they do well, we find that they do not get readmitted incessantly. When patients do not do well, and a fair number do not, then they are in the hospital a lot.

This is why we focused on patient selection; that is, saying who is going to be a good candidate. Can we figure this out before we go down the biventricular conversion pathway?

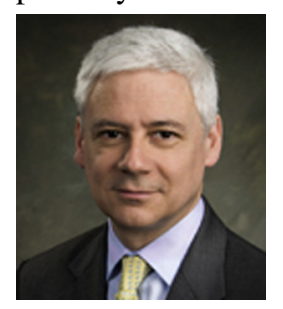

Dr Christian Pizarro (Wilmington, Del). A very nice presentation. I wonder if you could share with us among those patients who were lost, did you have the opportunity to actually get an autopsy and an examination of those hearts? Was there any residual EFE? Was there anything particular about the myocardium in those patients that could help us understand a little bit about this diastolic dysfunction?

Ms Herrin. Thank you for that question. It is an important point.

I do not know if any autopsy information was collected on these patients. I do know that sometimes at the time of EFE resection, samples are obtained that may lead to future analysis. I will defer this question as well. 
Dr Emani. I think that is a great point. The real Achilles heel of this operation is what we think is myocardial fibrosis. EFE is simply a marker for something underlying that I think both Dr McKenzie and you are bringing out. I think the key issue is how do we understand what is underneath the EFE? How do we understand the myocardium? I think even some unbalanced aortic valve canals may also have the same situation.

So we are trying to use magnetic resonance imaging techniques to help understand that ahead of time. We do not have those data for all of these patients, but we are hoping to be able to use either magnetic resonance imaging techniques or even some of the conductance catheter and left ventricle compliance measurements to assist.

We are also taking muscle biopsies at the time of left ventricle recruitment to get a sense of how the ultrastructural and histologic differences predict the ultimate outcome. I think that is where this is headed.

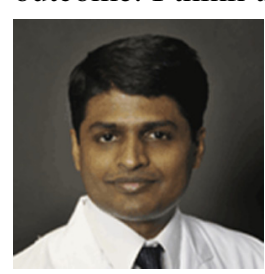

Dr T. K. Susheel Kumar (Memphis, Tenn). It seems in spite of all the preoperative studies and catheterization of patients you still had a $20 \%$ mortality rate. This is a huge problem when you try to convert single-ventricle to biventricular repair and they do not demonstrate favorable hemodynamic parameters. You have to make a quick decision to reconvert them back to single ventricle or you pretty much lose the patient.

Did you have a time point or did you have a decision tree as to how to salvage them back as a single ventricle or to let them be as such in the postoperative period and become candidates for heart-lung transplantation? How did you decide that?

Ms Herrin. Sure. Thank you for the question. As we have shown, elevated right ventricular pressure postoperatively can lead to poor outcomes, and so we certainly recommend monitoring these patients very closely.

In the event that we see elevated right ventricular pressure, I think again there may be reversible causes, and as Dr Emani said, often these patients undergo intervention, and that may be an option.

Watching them for a short period of time may be all right in some patients to see if the right ventricle pressure decreases, and that would require serial interval measurements.

But certainly, as you said, at some point the decision should be made to revert back to single ventricle or go down the ventricular assist device pathway, and I am not sure at this point that we have a recommended time for that. 